\title{
Computing $\mathrm{H}$-joins with application to 2-modular decomposition
}

\author{
Michel Habib • Antoine Mamcarz • \\ Fabien de Montgolfier
}

the date of receipt and acceptance should be inserted later

\begin{abstract}
We present here a general framework to design algorithms that compute $H$-join. For a given bipartite graph $H$, we say that a graph $G$ admits a $H$-join decomposition or simply a $H$-join, if the vertices of $G$ can be partitioned in $|H|$ parts connected as in $H$. This graph $H$ is a kind of pattern, that we want to discover in $G$. This framework allows us to present fastest known algorithms for the computation of $P_{4}$-join (aka $N$-join), $P_{5}$-join (aka $W$-join), $C_{6}$-join (aka 6 -join). We also generalize this method to find a homogeneous pair (also known as 2 -module), a pair $\left\{M_{1}, M_{2}\right\}$ such that for every vertex $x \notin\left(M_{1} \cup M_{2}\right)$ and $i \in\{1,2\}, x$ is either adjacent to all vertices in $M_{i}$ or to none of them. First used in the context of perfect graphs [Chvátal Sbihi 87], it is a generalization of splits (a.k.a 1-joins) and of modules. The algorithmics to compute them appears quite involved. In this paper, we describe an $O\left(m n^{2}\right)$-time algorithm computing all maximal homogeneous pairs of a graph, which not only improves a previous bound of $O\left(m n^{3}\right)$ for finding only one pair [Everett Klein Reed 97], but also uses a nice structural property of homogenous pairs, allowing to compute a canonical decomposition tree for sesquiprime graphs 1
\end{abstract}

Keywords algorithms $\cdot$ graph $\cdot$ graph decompositions $\cdot$ H-join $\cdot$ homogeneous pairs

\section{Introduction}

In the following, $G=(V, E)$ always denotes a simple, finite, undirected graph with $|V|=n$ vertices and $|E|=m$ edges. Two sets $X$ and $Y \subseteq V$ are said to be adjacent (resp. non adjacent) if $\forall x \in X \forall y \in Y, x y \in E$ (resp. $x y \notin E$ ). A vertex $x$ is said to be a splitter of $X \subset V$ if $\exists y, z \in X$ s.t. $x y \in E$ and $x z \notin E$.

LIAFA · CNRS · Université Paris Diderot - Paris 7

E-mail: \{habib, mamcarz, fm\}@liafa.univ-paris-diderot.fr

1 A preliminary version of this work was presented at Latin 2012 [15]. 
Let $H$ be a $l \times r$ matrix filled over $\{0,1\}$. We say that $G$ admits a $H$-join if $V$ can be partitioned into $l+r$ subsets $L_{1} \ldots L_{l}$ and $R_{1} . . R_{r}$ such that, for all $i \in[1 . . l]$ and all $j \in[1 . . r]$

- If $H[i, j]=1$ then for all $v \in L_{i}$ and for all $v^{\prime} \in R_{j} v v^{\prime} \in E$. In other words, there is a complete join (all possible edges exist) between $L_{i}$ and $R_{j}$.

- Else $(H[i, j]=0)$ then for all $v \in L_{i}$ and for all $v^{\prime} \in R_{j} v v^{\prime} \notin E$. In other words, there is no edge between $L_{i}$ and $R_{j}$.

We note $L=\bigcup_{i=1}^{i=l} L_{i}$ the left side of the $H$-join, and $R=\bigcup_{j=1}^{j=r} R_{j}$ the right side of the $H$-join. But sometimes $L$ and $R$ are identified with $\left\{L_{1} \ldots L_{l}\right\}$ and $\left\{R_{1} \ldots R_{r}\right\}$. Note that there are no constraints on the adjacency of two vertices on the same side of the join. The problem we deal with is:

\section{$H$-join Problem:}

Input: A graph $G=(V, E)$, and $H$ a $l \times r$ matrix filled over $\{0,1\}$.

Question: Does $G$ admit a $H$-join?

This problem was first defined under this name in [4,20, and it is a particular case of Matrix Partition problems as defined in [?]. Notice that equivalently, a bipartite graph $B_{H}$ may be used instead of $H$ to encode this problem. It is defined in the obvious way: $l$ white vertices $u_{1} \ldots u_{l}, r$ black vertices $v_{1} \ldots v_{r}$, and edge $u_{i} v_{j}$ iff $M_{i, j}=1$.

For a given $H$, a $H$-join is surjective if no set $R_{i}$ or $L_{j}$ is empty. The left (resp right) side is trivial if $\forall i\left|L_{i}\right| \leq 1$ (resp. if $\forall i\left|R_{i}\right| \leq 1$ ). The join is non-trivial if neither side is trivial.

Furthermore $H$ is twin-free if it has no two identical rows, nor two identical columns. Clearly, if two rows $i$ and $i^{\prime}$ are identical, then vertices of $G$ may be put indifferently in $L_{i}$ or in $L_{i^{\prime}}$. Using twins could be important in some application since this allows to formulate cardinality constraints, for the surjective case. In fact two identical rows $i$ and $i^{\prime}$ have a cardinality constraint meaning: $\left|V_{i} \cup V_{i^{\prime}}\right|$ must be at least 2 . Section 2.8 explains how to deal with these twins.

This formalism can be used to express a broad variety of problems, among which we can find connectedness of a graph ( $H$ is made up with 2 isolated vertices), but also many classical graph decompositions such as modular decomposition [16. Indeed, finding a module is equivalent to find a $H$-join in which $H$ is reduced to an edge and an isolated vertex. Using the same formalism one can search for 1-joins (aka splits) 8 ( $H$ is reduced to one edge and 2 isolated vertices one on each side), or 2-join ( $H$ is reduced to 2 parallel edges and 2 isolated vertices one on each side). For these decompositions, efficient ad-hoc algorithms exist. But for other decompositions like $P_{4}$-join, $P_{5}$-join, $C_{6}$-join, (see Figures $1,2,3$ ), this is the only known method. These decompositions were also defined and used in [5].

In this paper, we first propose a general technique for computing nontrivial $\mathrm{H}$-join in the surjective case and then we apply it to several particular 
cases, including the detection of homogenous pairs. In section 4 , focusing on this homogeneous pair decomposition we show how to compute a kind of tree decomposition for sesquiprime graphs using our techniques.

\section{Algorithms for the surjective H-join problem}

Let us now show, given $G$ and $H$, how to compute efficiently a surjective, non-trivial $H$-join.

\subsection{Seed sets}

In the following we will refer to the sets $L_{i}$ and $R_{j}$ as destination.

Definition 1 Let $H$ denotes an $l \times r$ matrix filled over $\{0,1\}$. A subset $S=$ $\left\{l_{1} \ldots l_{l}, r_{1} \ldots r_{r}\right\} \subseteq V$ is called a seed set.

If for given seed set $S$ and matrix $H$ we have that, for all $i \in[1 \ldots l]$ and $j \in[1 \ldots r] l_{i} r_{j} \in E \Longleftrightarrow H[i, j]=1$, then $S$ is compatible with $H$.

If there exists a seed set $S$ and a $H$-join $\left\{L_{1}, \ldots L_{l}, R_{1}, \ldots R_{r}\right\}$ such that for all $1 \leq i \leq l$ and $1 \leq j \leq r$ we have $l_{i} \in L_{i}$ and $r_{j} \in R_{j}$, then we say that $S$ realizes $H$ (or realizes this $H$-join) and that this $H$-join extends $S$.

It is easy to check if a seed-set is compatible with a matrix $H$. Clearly a seed-set realizing a $H$-join is compatible with $H$, but the converse is not true. Our brute force method to find H-decomposition is roughly the following:

1. Enumerate all possible seed sets,

2. For every generated seed set check if it could be extended as a H-join.

So there are two independent steps, the first one has to define how to efficiently generate all the possible seed sets and the second step is concerned by how to extend a seed set. Let us start by studying this first step.

\subsection{Generating the seed sets}

At first, it seems that we must consider $O\left(n^{l+r}\right)$ seed sets. However, we can low down a little this number, using techniques inspired from [10].

Proposition 1 If $G$ admits a $H$-join, every spanning tree of $G$ contains at least one edge of the $H$-join.

Proof The special case with $H$ filled with zeros can be trivially solved using any connected components algorithm. And if $H$ contains at least one 1 , it induces an edge cut of $G$, i.e. the join contains an edge of every path between vertices of $L$ and vertices of $R$. 
A spanning tree of $G$ contains $O(n)$ edges, including one that crosses the join. Therefore, by considering every edge of the spanning tree, and $l+r-2$ vertices, we have:

Proposition 2 It is enough to consider only $O\left(n^{l+r-1}\right)$ seed sets.

For some particular $H$ this bound can be improved as it is shown in the next sections.

2.3 Computing a $\mathrm{H}$-join that extends a given seed set

Let us first assume that $H$ has no false twin ( $H$ is twin-free for short).

Definition 2 Given $x \in V \backslash S, L_{i}$ is a potential destination of $x$ if for every $R_{j} \in R$, for every $y \in R_{j} x y \in E \Longleftrightarrow H[i, j]=1$. Dually $R_{j}$ is a potential destination of $x$ if for every $L_{i} \in L$, for every $y \in L_{i} y x \in E \Longleftrightarrow H[i, j]=1$.

Given a seed set $S$, for every $x \in V \backslash S$, let $\operatorname{list}(x) \subseteq\left\{L_{1}, \ldots, L_{l}, R_{1}, \ldots R_{r}\right\}$ denote the set of its potential destinations.

Proposition 3 Given a seed set $S$, for every $x \in V \backslash S$ there are at most two sets to which $x$ can belong. Moreover, at most one of these sets belongs to $R$, and at most one belongs to $L$.

Proof Since $H$ is twin-free, all neighborhoods in $H$ are different and therefore for every vertex $x \in V \backslash S$ at most one set $L$ (resp. $R$ ) is compatible with the adjacency to the vertices of $S$ that belong to $R$ (resp. $L$ ).

In other words, starting from the seed set $S, \operatorname{list}(x)$ has size at most 2 . If it has size 0 for some $x$ then that seed set cannot realize $H$. If it has size $1, x$ can safely be placed to the unique destination set it may belong to. It remains to address the case when $\operatorname{list}(x)$ has size 2 . In [13] the problem is solved using a 2-SAT solver 3]. Previous results, applied to homogeneous pairs, can be found in 12 using that idea.

Theorem 1 [13] Given a seed set $S$, it is possible to build a 2-SAT instance that extends $S$ to a $H$-join of $G$, if such partition exists, in $O\left(n^{2}\right)$ time.

Although 2-SAT can be solved using a linear time algorithm, the $n^{2}$ term in the complexity comes from the size of the 2-SAT instance which has $O\left(n^{2}\right)$ clauses. Furthermore, enumerating all possible seed sets yields an algorithm in $O\left(n^{|S|+2}\right)$ for computing a $\mathrm{H}$-join.

Now, we will show that it is possible to use partition refinement techniques in order to extend a seed set to a $H$-join, faster than the 2-SAT method. Partition refinement is a standard technique for graph algorithm design, see for example [19,17. 


\subsection{Destination rules}

Let us now give some Forcing Rules describing how a vertex can be placed to a destination $L_{i}$ or $R_{j}$. Later we shall explain how to implement them using partition refinement techniques. We say a vertex $x \in V$ is placed if $|\operatorname{list}(x)|=1$ (this is especially the case when $x \in S$ ) and ambiguous if $|\operatorname{list}(x)|=2$

Proposition 4 Let us consider a partial solution with placed vertices set into $\left\{L_{1} \ldots L_{l}, R_{1}, \ldots R_{r}\right\}$, and with all other vertices of $V$ ambiguous. Let $x$ be a placed vertex, and $y$ be an ambiguous vertex, such that list $(y)=\left\{L_{i}, R_{j}\right\}$,

1. If $\operatorname{list}(x)=\left\{L_{i^{\prime}}\right\}, H\left[i^{\prime}, j\right]=1$ and $x y \notin E$ then there exists no solution with $y$ placed to $R_{j}$

2. If list $(x)=\left\{L_{i^{\prime}}\right\}, H\left[i^{\prime}, j\right]=0$ and $x y \in E$ then there exists no solution with $y$ placed to $R_{j}$

3. If $\operatorname{list}(x)=\left\{R_{j^{\prime}}\right\}, H\left[i, j^{\prime}\right]=1$ and $x y \notin E$ then there exists no solution with $y$ placed to $L_{i}$

4. If $\operatorname{list}(x)=\left\{R_{j^{\prime}}\right\}, H\left[i, j^{\prime}\right]=0$ and $x y \in E$ then there exists no solution with $y$ placed to $L_{i}$

And if there is an $H$-join compatible with this partial solution, then there is also an $H$-join compatible with the partial solution in which $x$ has been placed to its destination.

Proof There are eight configurations depending on whether $x$ is on the left or right side, $H$ has an entry 0 or 1 , and $x y$ is an edge or not. For four of these configurations, $x$ adds no constraints to $y$. As for example the case in which: $\operatorname{list}(x)=\left\{L_{i^{\prime}}\right\}, H\left[i^{\prime}, j\right]=1$ and $x y \in E$.

Only the four cases listed in the proposition force $y$ to be placed. Let us consider the first case (the three other cases are similar): $x \in L_{i^{\prime}}, H\left[i^{\prime}, j\right]=1$ and $x y \notin E$. If $y \in R_{j}$ then the partial solution is not consistent with $H$, since $H\left[i^{\prime}, j\right]=1, x \in L_{j^{\prime}}$, and $x y \notin E$. So $y$ necessarily belongs to $L_{i}$.

So starting with a seed set $S$, we can first compute the lists, and then apply in any order these 4 forcing rules until no more can be applied. The partial solution grows, starting from $S$.

2.5 The extended partition refinement outline

Let us now consider how to efficiently compute the destinations of each vertex, using these forcing rules. There are two difficulties with them. First we must deal with edges but also with non edges, without having the complement graph. This can be handled by using vertex splitting, a standard partition refinement technique for graphs [19,17]. But sometimes we have to merge some classes. This is an extension of the standard partition refinement. Notice that termination is ensured because the merged classes are never split again, since they are exactly the destination sets, see below. 
Let us recall how vertex splitting works. For any partition $\mathcal{P}=\left\{P_{1}, \ldots P_{k}\right\}$ of the vertex set $V$ and any $A \subseteq V$, called a pivot-set, we define : Refine $(\mathcal{P}, A)$ $=\left\{P_{1} \cap A, P_{1}-A, \ldots, P_{k} \cap A, P_{k}-S\right\}$. The action of a pivot-set over the partition $\mathcal{P}$ (called refinement of $\mathcal{P}$ using $A$ ) is to create a new partition $\mathcal{P}^{\prime}=\operatorname{Refine}(\mathcal{P}, A)$ where each part $P \in \mathcal{P}$ is replaced with the two parts $P \cap A$ and $P \backslash A$. However empty parts are not inserted in $\mathcal{P}^{\prime}$. A part $P$ is thus splitted only if $P \not \subset A$ and $P \cap A \neq \emptyset$. For various graph applications $A=N(x)$ for some pivot-vertex $x \in V$, and therefore the current partition is refined using some neighborhood.

Lemma 1 [17] If a graph $G$ is given by its adjacency lists, for every partition $\mathcal{P}$ of $V$, Refine $(\mathcal{P}, N(x))$ and Refine $(\mathcal{P}, \overline{N(x)})$ can be computed in $O(|N(x)|)$.

So given a graph $G$, partition refinement techniques allow to compute dually (i.e., either on $G$ or $\bar{G}$ ) within the same complexity. Before going into the details of the algorithm let us now sketch it, in 3 main steps:

1. Given a seed set, compute the list of every vertex of $V$.

This can be obtained using standard partition refinement, starting from the initial trivial partition $\mathcal{P}=\{V\}$ using successively the neighborhood of every vertex of $S$ as pivot. Then Procedure GENLISTS (see 2.6.1) assigns the lists to each part. See Section 2.6.1 for details. After this step we have a partition that is a partial solution, with nonempty destination sets containing all placed vertices. A part is a set of vertices with the same list.

2. Apply all possible forcing rules.

To avoid generating trivial $H$-join, one more placed vertex is needed. If all vertices of $V \backslash S$ are ambiguous, the algorithm tries the two possible affectations of this given vertex $v_{0}$ to start the process. Note that if a solution exists, at least one of these affectations will extend into a nontrivial $H$-join.

Finally Algorithm 2 implements Proposition 4 recursively, using a queue $F$ which is the queue of the newly placed vertices that may help to affect other ambiguous vertices. Using $F$ allows us to find the pivot vertices in constant time.

3. Test whether the current partition can be extended as a solution. When there are no more placed vertices that can be used as pivots, the process stops. Proposition 5 tells that all remaining ambiguous vertices can be put on one side of the join. We process using necessary conditions, so if the seed extends to a $H$-join, then the computed partition is a $H$-join. That must be finally checked. This test can be done in $O(n+m)$ time using a straightforward algorithm.

\subsection{Algorithm details}

Let us now detail the implementation. For our unconventional partition refinement we need, in addition to procedure refine(), some particular procedures. 
The procedure move_vertex $(\mathcal{P}, x, P)$ extracts $x$ from its current part of $\mathcal{P}$ and put it into part $P$. It returns the new partition. Procedure merge $(\mathcal{P}, P, Q)$ return a new partition in which $P \cup Q$ is a new part, replacing both $P$ and $Q$.

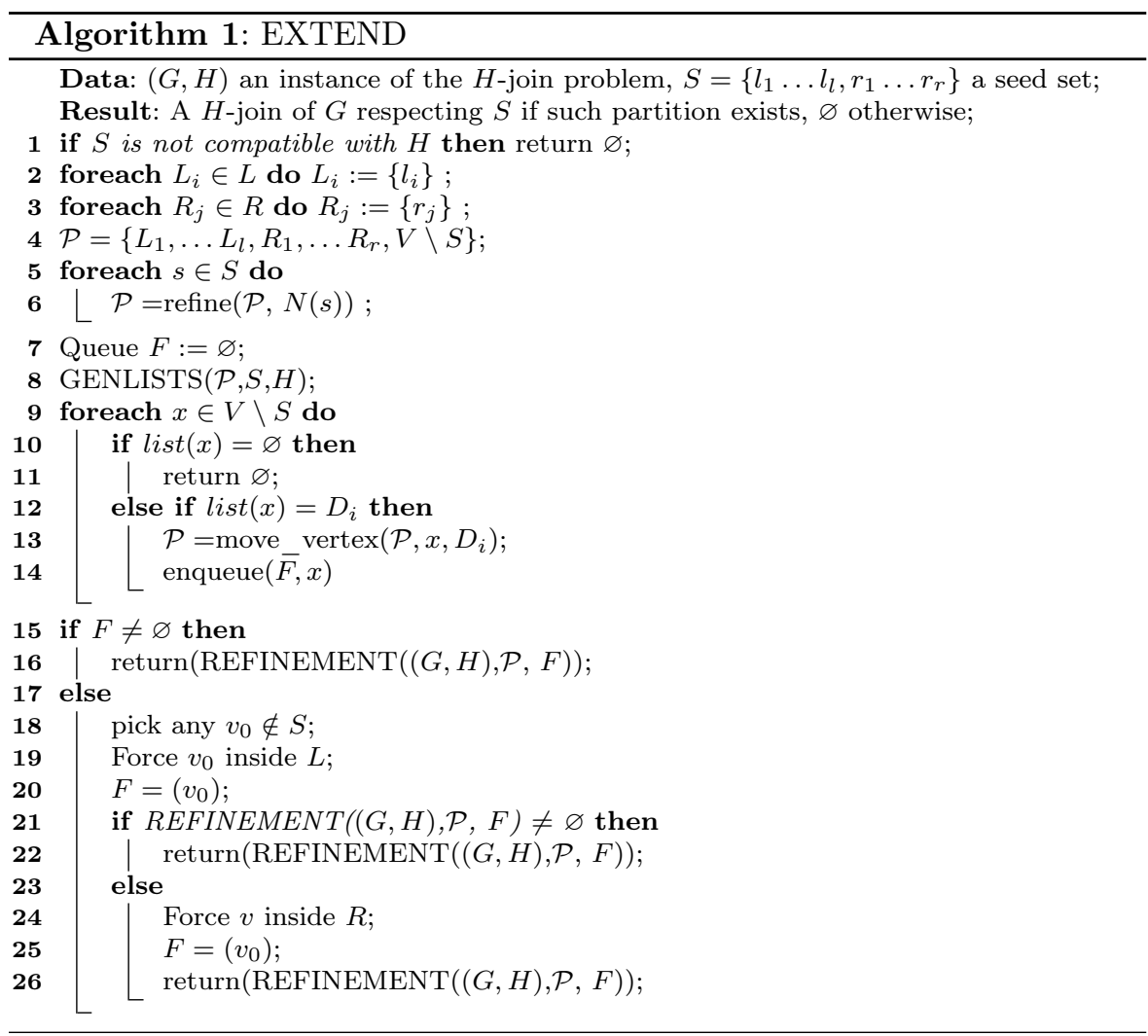

\subsubsection{Procedure GENLISTS}

Procedure $\operatorname{GENLISTS}(\mathcal{P}, S, H)$ computes $\operatorname{list}(P)$ for each part $P \in \mathcal{P}$ created after the $|S|$ initial refinements. We can assume that each part $P$ has a neighbors list and, if a new part $P \cap N(x)$ is created during refinement, (while the old $P$ becomes $P \backslash N(x)$ ), then $x$ is added to a neighbors list of the new part which inherits (in constant time using linked lists with a common tail, i.e., an in-tree) from the neighbors list of the former $P$ part. The neighbors list of the parts allows to build the lists. Indeed if we use the pivots in order $l_{1}$ then $l_{2} \ldots$ then $l_{l}$ then $r_{1} \ldots$ then $r_{r}$; and if the new part $P \cap N(x)$ is put just before (in a total ordering of parts) the old part $P \backslash N(x)$, then all parts are sorted in lexicographic order with respect to $S$. We can sort matrix $H$ in the same order. 


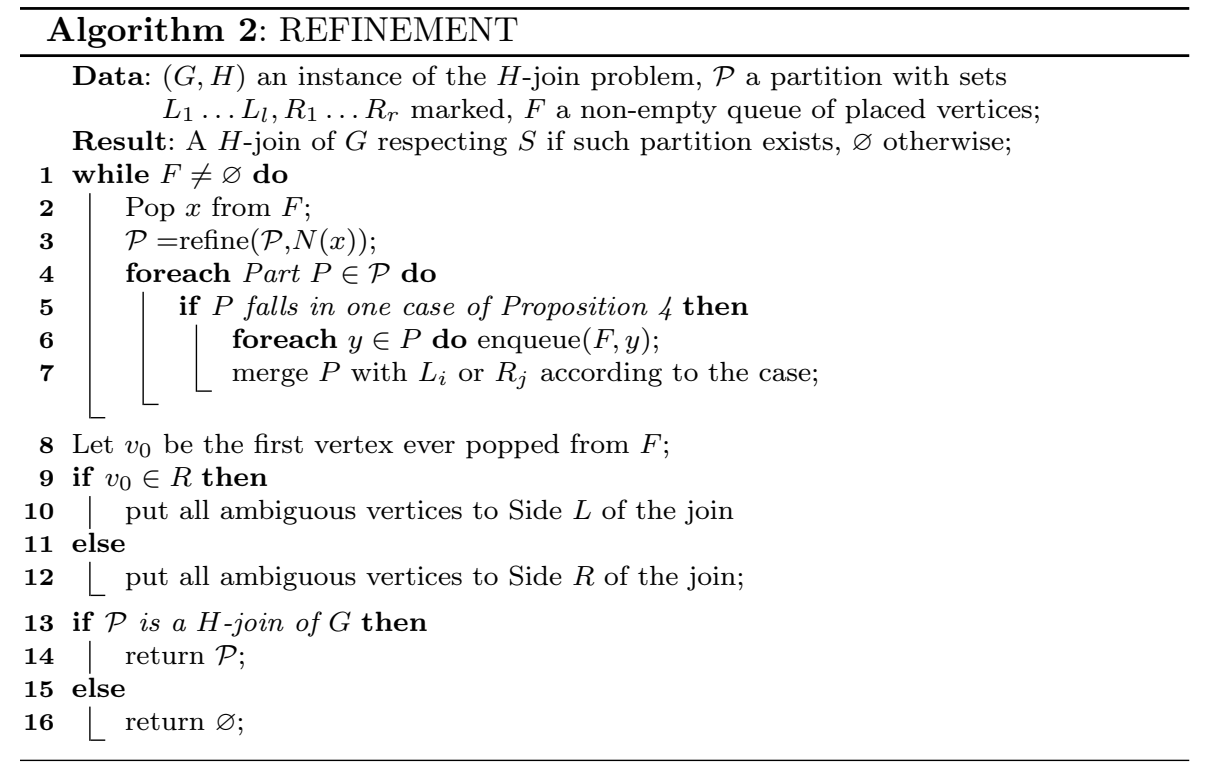

Therefore, for each part $P$, if it corresponds with one (and at most one since $H$ is twin-free) $R_{j}$, then $R_{j}$ is placed to list $(P)$, i.e. with this implementation, all vertices $x \in P$ implicitly get $R_{j}$ to their list. This is done in one sweep with $j$ from 1 to $r$.

The same is then done for the right side also (affect $L_{i}$ to $\operatorname{list}(P)$ ) after dropping $l_{1} \ldots l_{l}$ from the neighbors lists and re-sorting them in lexicographic order (taking linear time).

\subsection{Correctness of the EXTEND algorithm}

Proposition 5 In the execution of EXTEND algorithm, when $F$ becomes empty,

- either all remaining ambiguous vertices can be put into $R$ (right side)

- or into $L$

Proof Let us prove it only for the $R$ side (the proof for the other side is the same). Let $P$ be a part of ambiguous vertices with $\operatorname{list}(P)=\left\{L_{i}, R_{j}\right\}$. We affect all vertices from $P$ to $R_{i}$. For all vertices currently in the left side, the constraints of $H$ are respected (if not, some vertex would not have been ambiguous by proposition 3 , and would have been inserted into $F$ ). Since we do not add further vertices to this side, so those constraints remain respected.

Theorem 2 The Refinement Algorithm 2, given an instance $(G, H)$ and a seed $S$ of $r+l$ vertices, computes a non-trivial $H$-join $\mathcal{P}$ that extends $S$ if such $H$-join exists, and returns $\varnothing$ otherwise. 
Proof Correctness of the main loop follows from Proposition 4 If after step 2 there are no more ambiguous vertices, then the necessary conditions of Proposition 4 tell that $\mathcal{P}$ is the only $H$-join possible extending $S$ (provided $\mathcal{P}$ is a genuine $H$-join, what is ultimately tested by the algorithm). In the other case, by Proposition 3, every ambiguous vertex has a list of size 2, with one destination for each side of the join. By Proposition 5. putting all ambiguous vertices on each side of the partition gives $H$-join extending $S$ (again, if possible). To be sure that the $H$-join is not trivial, if $v_{0}$, the first pivot used after the vertices from $S$, is on the left side, then all ambiguous are put on the right side, and vice versa.

2.8 Dealing with twins

Proposition 6 Let $(G, H)$ be an instance of the $H$-join problem, and $S$ a seed set compatible with $H$. It is possible to build $H^{\prime}$ such that $H^{\prime}$ is twin-free, and that $S$ realizes $H$ if and only $S$ is realizes $H^{\prime}$.

Proof Let $H^{\prime}$ be $H$ in which every twin class has been contracted. In this case, $S$ contains more than one vertex for each destination (in fact, one for each twin in the twin class).

Take any solution to the $H^{\prime}$ partition problem. Simply split the previously merged destinations. Since there are no constraints between those new sets, and since there is enough vertices in $S$ to do so, this is always possible.

Conversely, consider any solution to the $H$-join problem. Assume $D$ and $D^{\prime}$ are twins in $H$, just merge $D$ and $D^{\prime}$ to obtain a solution to the $H^{\prime}$-join problem.

\subsection{Complexity issues}

Proposition $7 \mathcal{P}$ always contains at most $2 r+2 l+r l$ parts

Proof We create an initial partition with $|S|+1$ parts but only one nontrivial part $V \backslash S$, and then perform $|S|$ pivoting on it. Theoretically this steps can create $O\left(|S|+2^{|S|}\right)$ parts. After Procedure $\operatorname{GENLISTS}(\mathcal{P}, S, H)$ creates lists $(P)$ for each class $P$, two parts cannot have the same list since $H$ is twinfree. As $\operatorname{list}(P)$ cannot be empty either, then we have at most $l$ parts with lists $(P)=\left\{L_{i}\right\}$; at most $r$ parts with lists $(P)=\left\{R_{j}\right\}$; and at most lr parts with $\operatorname{lists}(P)=\left\{L_{i}, R_{j}\right\}$. Plus the $|S|$ parts $L_{1} \ldots L_{l}$ and $R_{1} \ldots R_{r}$. All other parts have an empty list, thus if in the initialization step creates more than $2 r+2 l+r l$, we can end with $\operatorname{list}(P)=\varnothing$ and the algorithm stops.

When a refine is done by Algorithm 2 then Proposition 4 ensure that one of the two classes issued from a part $P$, either $P \cap N(x)$ or $P \backslash N(x)$, is merged with one of its destination set $\left(L_{i}\right.$ or $\left.R_{j}\right)$. Therefore the number of parts cannot increase and is bounded by $2 r+2 l+r l$. 
Proposition 8 Algorithm 1 requires $O(n l r+m)$ time.

Proof Lemma 1 tells that $\operatorname{refine}(\mathcal{P}, x)$ runs correctly and in $O(|N(x)|)$ time (the standard implementation is that vertices from $N(x)$ are moved to newly created parts $P \cap N(x)$, while the unmoved vertices stay in the old parts $P \backslash N(x)$ needing no operation). Since each neighborhood is used at most one time as a pivot, the time taken by all the calls to refine is $O(m)$.

By using lists to store every destination $L_{i}$ or $R_{j}$, each merging step can be done in constant time, which implies that all merging will take $O(n)$ time (since every vertex is moved only one time). There are also $O(n)$ queue operations (each vertex is put at most once into $F$ ).

Procedure $\operatorname{GENLISTS}(\mathcal{P}, S, H)$ takes $O(n+|S|)$ time since it uses lineartime lexicographic sorting, and simultaneous sweep of two sorted lists.

The only problem we may have is the line 2 of Algorithm 2. We have to check every part $P$ to see if its vertices fall in one case of Proposition 4 . This check is done in constant time per part, and Proposition 7 insures that the number of parts is always bounded by $2 r+2 l+r l$.

Let $h$ denotes the number of ones in the $H$ matrix.

Theorem 3 There is a $O\left(n^{l+r-1} m l r h\right)$ time algorithm for $H$-join problem, i.e. that produces a surjective, non-trivial $H$-join if possible.

Proof First assume $G$ is connected. Generate the seed $S$ by taking one edge among the $n-1$ of the spanning tree, and $n-2$ other vertices of $G$. Identify the edge with every 1 entry of matrix $H$ (at most $h$ of them). Then, use the refinement procedure presented above. And if $G$ is not connected, all connected components except one shall be put in one destination set, so work component by component.

Corollary 1 If $H$ is not considered to be a part of the input, there is a $O\left(n^{l+r-1} m\right)$-time algorithm to solve the H-Join problem.

\section{An almost-surjective case}

\subsection{Algorithms}

The algorithm for the surjective case above may be extended to a restricted class of non-surjective case, when we allow two "trash sets" that may be empty. Each trash set $R_{1}$ or $L_{1}$ is non-adjacent with the other side of the join. This class includes almost all known $H$-join problem people are looking at.

More formally, given $H$, we say that $G$ admits an Almost Surjective $H$-join (ASH-join) if $G$ admits a $H$-join $\mathcal{P}=\left\{L_{1} \ldots L_{l}, R_{1} \ldots R_{r}\right\}$ such that

- For all $i \in 2 \ldots l L_{i}$ contains at least one vertex.

- For all $i \in 2 \ldots r R_{i}$ contains at least one vertex.

- $R_{1}$ is non adjacent to every $L_{i}$, i.e. $\forall i H[i, 1]=0$ 
- $L_{1}$ is non adjacent to every $R_{j}$, i.e. $\forall j H[1, j]=0$

- $L=\cup L_{i}$ contains at least $l$ vertices

- $R=\cup R_{j}$ contains at least $r$ vertices.

Define an AS-seed set as a set $\left\{l_{2} \ldots l_{l}, r_{2} \ldots r_{l}\right\}$. The same definition of a compatible seed and a seed that realizes $H$ apply. Then, the proofs and algorithms go on as in the surjective case. We only need to prove those two propositions:

Proposition 9 Given a AS-seed set $S$, for every $x \in V \backslash S$ there are at most two sets to which $x$ can belong to. Moreover, at most one of these sets belongs to $R$, and at most one belongs to $L$.

Proof Notice that $L_{1}\left(R_{1}\right)$ is uniformly adjacent to $R(L)$. Since $H$ is twin-free, every pair of vertices of $L(R)$ admits a splitter in $R_{2} \ldots R_{r}\left(L_{2} \ldots L_{l}\right)$, thus at most one vertex of $L(R)$ can respect the adjacency to the vertices of $S$ that belong to $R(L)$

Proposition 10 If $G$ admits a ASH-join, every spanning tree of $G$ will contain at least one edge between to non-empty sets of the $H$-join.

Proof Since $R_{1}$ and $L_{1}$ are non-adjacent to the other side of the join, no edge of the cut can be adjacent to them. All the remaining sets are non-empty.

Finally we have:

Theorem 4 There is a $O\left(n^{l+r-3} * l r * m\right)$ time algorithm for ASH-join problem.

\subsection{Applications}

Let us now briefly describe three applications to known $H$-join problems for which our general framework provides the fastest known algorithms. Notice that our general procedure provides an algorithm in $O(\mathrm{~nm})$ to find a 1-join, which is slower than state-of-the-art algorithms that provide the whole decomposition tree in linear time [8].

It may also be applied to 2-join which is an almost surjective $H$-join problem, and provides an algorithm in $O\left(n^{3} m\right)$ which, again, is slower than stateof-the-art $O\left(n^{2} m\right)$ algorithms [10].

\subsection{1 $P_{4}$-join}

A graph is said to admit a $P_{4}$-Join (or $N$-join) if its vertex set can be partitioned into six sets $R_{0}, R_{1}, R_{2}, L_{0}, L_{1}, L_{2}$ as depicted in Figure 1 (an edge $L_{i} R_{j}$ appears if $H[i, j]=1$, i.e $L_{i}$ must be completely adjacent with $R_{j}$, and no edge is drawn if $H[i, j]=0$, i.e no edge between $L_{i}$ and $R_{j}$ is allowed) [5].

Corollary 2 There is an $O\left(m n^{3}\right)$ time algorithm to compute a non-trivial $P_{4}$-join if exists any.

Proof This decomposition is quasi-surjective and we can apply theorem 4 which yields an $O\left(m n^{3}\right)$ time algorithm. 
Fig. 1 Structure of a $P_{4}$-Join

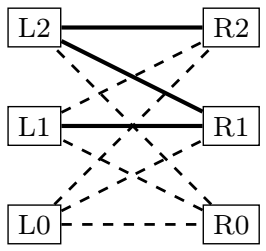

Fig. 2 Structure of a $P_{5}$-Join

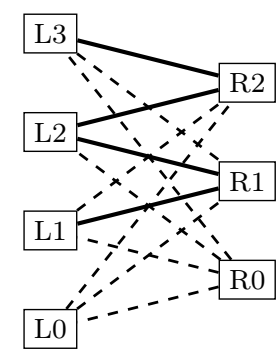

3.2.2 $P_{5}$-join

A graph is said to admit a $P_{5}$-Join (or $W$-join) if its vertex set can be partitioned into seven sets $R_{0}, R_{1}, R_{2}, L_{0}, L_{1}, L_{2}, L_{3}$ as depicted in Figure 2 (an edge $L_{i} R_{j}$ appears if $H[i, j]=1$, i.e $L_{i}$ must be completely adjacent with $R_{j}$, and no edge is drawn if $H[i, j]=0$, i.e no edge between $L_{i}$ and $R_{j}$ is allowed) [5]. It is a particular case of quasi-surjective $\mathrm{H}$-join and therefore:

Corollary 3 There is a $O\left(m n^{4}\right)$ time algorithm to compute a non-trivial $P_{5}$ join, if exists any.

\subsection{3 $C_{6}$-join}

The $C_{6}$-join was introduced by 2, 6, under the name of 6-join, to decompose some perfect graphs. A graph is said to admit a $C_{6}$-Join if its vertex set can be partitioned into 8 sets $L_{0} \ldots L_{3}$ and $R_{0} \ldots R_{3}$ as depicted in Figure 3 . It is a particular case of quasi-surjective $\mathrm{H}$-join and therefore:

Corollary 4 There is a $O\left(m n^{5}\right)$ time algorithm to compute a non-trivial $C_{6}$ join of a graph, if exists any.

\section{Homogeneous Pairs}

First used in the context of perfect graphs [11, homogeneous pairs generalize splits (a.k.a 1-joins) and modules. The algorithmics to compute them appears 
Fig. 3 Structure of a $C_{6}$-Join

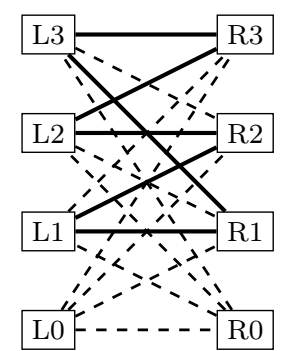

quite involved. In the following, we describe an $O\left(m n^{2}\right)$-time algorithm computing (if any) a homogeneous pair, which not only improves a previous bound of $O\left(m n^{3}\right)$ [12, but also uses a nice structural property of homogenous pairs.

\subsection{Definition}

A homogeneous pair of $G$ is a set $P \subseteq V$ which can be partitioned into two subsets $M_{1}$ and $M_{2}$ such that neither $M_{1}$ nor $M_{2}$ has a splitter outside $P$ (i.e. all splitters of $M_{i}$ belong to $M_{3-i}$ ).

If $2<|P|<|V|-1, P$ is called a proper (or non trivial) homogeneous pair. The definition implies that, if such a proper pair exists, the whole vertex-set is partitioned into six sets $M_{1}, M_{2}, A, B, C, D$, such that $|A|+|B|+|C|+|D|>2$ and $\left|M_{1}\right|+\left|M_{2}\right|>2$ and there are 4 adjacencies (i.e., complete bipartite, drawn as plain lines) and four non-adjacencies (dotted lines) between the sets as depicted in Figure 4 (no edge is drawn if any linkage between sets is allowed). In the following, we will sometime refer to vertices or subsets of $P$ as inner, and to vertices or subsets of $V \backslash P$ as outer. Clearly, the inner and outer sides are the two sides of a $\mathrm{H}$-join.

Fig. 4 Relationships between a homogeneous pair and the other vertices

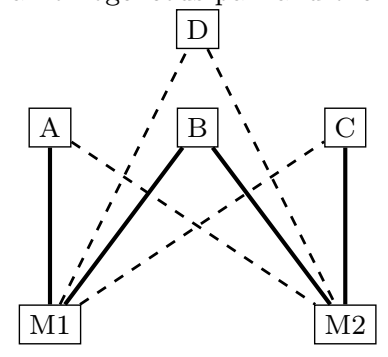

Even though homogeneous pair detection is not a $\mathrm{ASH}$-join problem, we can generalize a bit further our method to solve it. 
Let $\bar{G}$ denote the edge-complement of $G$. Immediately from the above definition, by swapping $A$ and $C$, and also $B$ and $D$, we have:

Proposition $11 P$ is a homogeneous pair of $G$ iff $P$ is a homogeneous pair of $\bar{G}$.

Remark 1 A homogeneous pair with $M_{1}=\varnothing$ or $M_{2}=\varnothing$ or $A=C=\varnothing$ is a homogeneous set (also called module).

We say a module is trivial if it has 0,1 or $n$ vertices. A graph is prime if all its modules are trivial ones.

Homogeneous pairs were used in [1] in the context of perfect graphs. They form a natural generalization of splits (in this case $M_{1}$ has no neighbors in $V$ $\left.\left(M_{1} \cup M_{2}\right)\right)$ and of modules (in this case $M_{1}$ is empty). Linear-time algorithms for computing the split decomposition [8] or the modular decomposition (see [16] for a survey) are known, but are quite involved. An $O\left(m n^{3}\right)$ algorithm was proposed in [12] and it is therefore a challenging problem to design an efficient algorithm to find homogeneous pairs. Modules of graphs can be represented via a directed tree, using the partitive families framework, see [9, while splits can be represented via an undirected tree through bipartitives families [7, 18]. Unfortunately the structure of homogeneous pairs seems to be weaker than the one of splits. Nevertheless, we can prove a tree structure theorem.

Definition 3 A sesqimodule (introduced in [18]) is a set $M$ such that there exists $x$ such that $G[V \backslash\{x\}]$ is a module. It is proper if $|M|>1$.

A graph is sesquiprime if it contains no sesquimodule.

The family of sesquimodules is thus decomposed into modules (if $M$ is a module any $x$ is $\mathrm{OK}$ ) and homogeneous pairs $\{M,\{x\}\}$ where $x$ is the only splitter of $M$. Notice that in this case $M$ is also a homogeneous pair $\{M \cap N(x), M \backslash$ $N(x)\}$. So sesquimodules are special cases of homogeneous pairs, so named because they stand between the usual 1-modules and the 2-modules.

A sesquiprime graph is also prime (it contains no proper module).

Proposition 12 All the sesquimodules of a graph can be enumerated in time $O(n m)$.

Proof For each $x \in V$ just run a linear-time modular decomposition algorithm on $G[V \backslash\{x\}]$

Since when the graphs contains a sesquimodule, it is easy to find a homogeneous pair, we will only address the case of sesquiprime graphs.

4.2 Structural properties of homogeneous pairs

Lemma 2 Let $G$ be a sesquiprime graph and $P$ be a proper homogeneous pair of $G$. There exists only one way to partition $P$ into $\left\{M_{1}, M_{2}\right\}$. 
Proof Consider a partition $\left\{M_{1}, M_{2}\right\}$ of $P$ with $m_{1} \in M_{1}$ ang $m_{2} \in M_{2}$. Assume, by contradiction, that it exists another split $\left\{M_{1}^{\prime}, M_{2}^{\prime}\right\}$ of $P$ with $m_{1}$ and $m_{2}$ in the same subset (w.l.o.g., $M_{1}^{\prime}$ ). By definition of a homogeneous pair, $m_{1}$ and $m_{2}$ have the same neighborhood outside $P$, which implies that $A$ and $C$ were empty for the partition $\left\{M_{1}, M_{2}\right\}$, i.e., that $P$ is a homogeneous set, contradicting primality of $G$.

The graphs we consider in the following are assumed to be sesquiprime, unless explicitly stated otherwise. Among other interesting properties discussed below, we gain then that it is equivalent to talk about $P$ or about $\left\{M_{1}, M_{2}\right\}$ when dealing with a homogeneous pair. And that is why $P$, a single subset, may be called a "pair".

A proper homogeneous pair $P$ is maximal (resp. minimal) if there is no proper homogeneous pair $Q$ such that $P \subsetneq Q($ resp. $Q \subsetneq P)$.

Remark 2 A homogeneous pair of a graph that induces a minimal set of inner vertices (resp. outer) vertices induces a maximal set of outer (resp. inner) vertices.

Let us now consider the relationships between homogeneous pairs.

Theorem 5 Let $G$ be a sesquiprime graph, $P=\left\{M_{1}, M_{2}\right\}$, and $P^{\prime}=\left\{M_{1}^{\prime}, M_{2}^{\prime}\right\}$ be two proper homogeneous pairs of $G$. Then

1. either $P \cap P^{\prime}=\varnothing$

2. or $P \cap P^{\prime}=\{x\}$ and $x$ is not a splitter of $M_{1} \backslash\{x\}$ nor $M_{2} \backslash\{x\}$ nor $M_{1}^{\prime} \backslash\{x\}$ nor $M_{2}^{\prime} \backslash\{x\}$ (i.e. both $P-\{x\}$ and $P^{\prime}-\{x\}$ are homogeneous pairs).

3. or $P \cup P^{\prime}$ is a homogeneous pair

Proof Consider the four intersection sets $M_{i} \cap M_{j}^{\prime}$ for $i, j=1,2$. We distinguish four cases.

1. All these four intersection sets are empty. Then we are in Case 1.

2. Exactly three intersection sets are empty. Suppose without loss of generality $M_{1}$ properly intersects $M_{1}^{\prime}$. We first prove $M_{1} \cap M_{1}^{\prime}$ is a module. Let $y$ be a splitter of $M_{1} \cap M_{1}^{\prime}$. If $y \notin P, y$ would split $M_{1}$ but $P$ is a homogeneous pair, a contradiction. If $y \notin P^{\prime}, y$ would split $M_{1}^{\prime}$ but $P^{\prime}$ is a homogeneous pair, a contradiction. So $y \in P \cap P^{\prime}$ but $P \cap P^{\prime}=M_{1} \cap M_{1}^{\prime}$ : $y$ is not a splitter, final contradiction. As $G$ is prime $M_{1} \cap M_{1}^{\prime}$ is trivial, i.e. $M_{1} \cap M_{1}^{\prime}=\{x\}$ : this is Case 2 of the theorem. As $G$ is sesquiprime then $M_{1}$ also contains $u \neq x$ and $M_{1}^{\prime}$ also contains $u^{\prime} \neq x$.

Suppose $x u$ is an edge. Then $u u^{\prime}$ is an edge (otherwise $u$ is a splitter of $M_{1}^{\prime}$ ) and then $u^{\prime} x$ is an edge (otherwise $u$ is a splitter of $M_{1}$ ). Finally $x$ is adjacent with every vertex of $M_{1} \cup M_{1}^{\prime}$, and is not a splitter of $M_{1}-\{x\}$ nor of $M_{1}^{\prime}-\{x\}$. And if $x u$ is not an edge, $x$ is non-adjacent to $M_{1} \cup M_{1}^{\prime}$ and is not a splitter either.

Suppose $x$ is a splitter of $M_{2}$ : there exists $y, z \in M_{2}$ and $x y$ is an edge but not $x z$. Since $y$ does not split $P^{\prime} y u^{\prime}$ is an edge, while $z u^{\prime}$ is not an 
edge for the same reason. So $u^{\prime} \notin P$ is a splitter of $M_{2}$, contradiction with $P$ is a homogeneous pair. For the same reason $x$ is not a splitter of $M_{2}^{\prime}$. Consequently, if $|P|>3$ and $\left|P^{\prime}\right|>3$, then $P-\{x\}$ and $P^{\prime}-\{x\}$ are homogeneous pairs.

3. Suppose now exactly two intersection sets are non empty. We must distingish subcases:

(a) Suppose $M_{1}$ intersects $M_{1}^{\prime}$, and $M_{2}$ intersects $M_{2}^{\prime}$. Then $P \cup P^{\prime}$ is a homogeneous pair $\left\{M_{1} \cup M_{1}^{\prime}, M_{2} \cup M_{2}^{\prime}\right\}$. Indeed for $u_{1} \in M_{1} \cap M_{1}^{\prime}$ and $x \notin P \cup P^{\prime}$, if $x u_{1}$ is an edge $x$ is adjacent with both $M_{1}$ and $M_{1}^{\prime}$ (otherwise it would split $M_{1}$ or $M_{1}^{\prime}$ ) and thus does not split $M_{1} \cup M_{1}^{\prime}$. And if $x u_{1}$ is not an edge then $x$ is non-adjacent with $M_{1} \cup M_{1}^{\prime}$. This is Case 3 of the theorem. A similar proof shows $x$ is not a splitter of $M_{2} \cup M_{2}^{\prime}$.

(b) Suppose $M_{1}$ intersects $M_{2}^{\prime}$, and $M_{2}$ intersects $M_{1}^{\prime}$. Then $P \cup P^{\prime}$ is a homogeneous pair $\left\{M_{1} \cup M_{2}^{\prime}, M_{2} \cup M_{1}^{\prime}\right\}$ (same proof than 3(a))

(c) Suppose $M_{1}$ intersects both $M_{1}^{\prime}$ and $M_{2}^{\prime}$, but $M_{2}$ does not intersect $P^{\prime}$. Then $P \cup P^{\prime}$ is a homogeneous pair $\left\{M_{1} \cup P^{\prime}, M_{2}\right\}$. Indeed let $u_{1} \in M_{1} \cap M_{1}^{\prime}$ and $u_{2} \in M_{1} \cap M_{2}^{\prime}$. Let $x \notin P \cup P^{\prime}$. Suppose $x u_{1}$ is an edge. As $x$ is not a splitter of $M_{1} x$ is adjacent with $M_{1}$. So $x u_{2}$ is an edge. As $x$ is not a splitter of $M_{1}^{\prime}\left(\operatorname{resp} . M_{2}^{\prime}\right) x$ is adjacent with $M_{1}^{\prime}$ (resp. $M_{2}^{\prime}$ ). As similarly if $x u_{1}$ is not an edge $x$ is non-adjacent with $M_{1} \cup P^{\prime}$. This is also Case 3 of the theorem.

(d) The three remaining cases are similar with $3(\mathrm{c})$ if $M_{1}$ is replaced by $M_{2}$, or if we swap $P$ and $P^{\prime}$, or both.

4. Suppose now at least three intersection sets are nonempty. Then either Case 3(a) or Case 3(b) holds. Suppose w.l.o.g $M_{1}$ intersects $M_{1}^{\prime}$, and $M_{2}$ intersects $M_{2}^{\prime}$. As seen before $\left\{M_{1} \cup M_{1}^{\prime}, M_{2} \cup M_{2}^{\prime}\right\}$ is a homogeneous pair. But a third intersection holds! If $M_{1}$ intersects also $M_{2}^{\prime}$ then a neighbor (resp. non-neighbor) of $M_{1} \cup M_{1}^{\prime}$ also is a neighbor (resp. non-neighbor) of $M_{2} \cup M_{2}^{\prime}$ and $P \cup P^{\prime}$ is a module. As $G$ is sesquiprime, $P \cup P^{\prime}=V$. Same if $M_{1}^{\prime}$ intersects also $M_{2}^{\prime}$. It is a degenerated form of Case 3 of the theorem.

4.3 Homogeneous pairs decomposition

The modules of a graph are closed under union: for them only Cases 1 or 3 of Theorem 1 may occur. But Theorem 5 tells the homogeneous pairs are almost closed under union, in the following sense. Let us say that a vertex $x$ is a conflicting vertex of a homogeneous pair $P$ if there exist a homogeneous pair $P^{\prime}$ such that $P \cap P^{\prime}=\{x\}$.

Proposition 13 A proper homogeneous pair $P$ of a sesquiprime graph has size at least 4 and contains at most two conflicting vertices. 
Proof Let $P=\left\{M_{1}, M_{2}\right\}$. If $\left|M_{1}\right|=0 M_{2}$ is a module, contradicting primality, thus sesquiprimality, of $G$. And if $\left|M_{1}\right|=1 P$ is a sesquimodule, also impossible. Same for $M_{2}$. So $P$ has at least four vertices.

As a consequence of Case 2 of Theorem 5, a conflicting vertex $x$ is either fully adjacent with $M_{i}$ or fully non-adjacent with $M_{i}$, for $i=1$ or 2 . Furthermore $x$ belongs either to $M_{1}$ or to $M_{2}$. There are thus eight cases. All vertices falling in the same case form a module, and since the graph is prime there is at most one vertex per case. Suppose $M_{1}$ contains two conflicting vertices $x$ and $y$. If $x y \in E$ both $x$ and $y$ must be fully adjacent with $M_{1}$. And if $x y \notin E$ both $x$ and $y$ must be fully non-adjacent with $M_{1}$. Since there are no twins in $G, x$ has to be fully adjacent with $M_{2}$, and $y$ fully non-adjacent with $M_{2}$. So $M_{2}$ does not contains a conflicting vertex, and $M_{1}$ can not contain a third conflicting vertex (it would be a twin of $x$ or $y$ ). The same proof shows that if $M_{2}$ contains two conflicting vertices then there are exactly two in $M_{2}$ and zero in $M_{1}$.

Definition 4 A vertex is a maximal conflicting vertex if it is the conflicting vertex of two maximal (wrt inclusion) homogeneous pairs of $G$.

A almost maximal homogeneous pair (AMHP for short) of a graph $G$ is obtained by taking a homogeneous pair of $G$ maximal with respect to inclusion, ad removing its (at most two) conflicting vertices.

Theorem 6 - Every vertex of $G$ either belongs to a unique AMHP, or is a maximal conflicting vertex.

- Each proper homogeneous pair P of $G$ intersects exactly one almost maximal homogeneous pair Max $(P)$.

- Furthermore a proper homogeneous pair $P$ is included in exactly one maximal homogeneous pair, namely the $\operatorname{Max}(P)$ plus its (at most two) conflicting vertices.

Proof Consequence of Theorem 5 and of Proposition 13

This theorem allows to compute a canonical (i.e., independent from algorithms choices) homogeneous pair decomposition tree of a sesquiprime graphs. Let the almost maximal homogeneous pairs partition (AMHPP for short) of a graph the partition of the vertex-set into almost-maximal homogeneous pairs and into maximal conflicting vertices. The existence and uniqueness of this partition is ensured by the previous theorem. A tree may then be built by recursively computing the AMHPP inside the graph induced by each AMHP. The next section shows how to compute this AMHPP efficiently.

\section{Homogeneous pairs algorithm}

5.1 Finding a Homogeneous pair

Definition 5 A $H P$-seed-set is a triple $\left\{m_{1}, m_{2}, a\right\}$ of vertices of $V$ such that $m_{1} \in M_{1}, m_{2} \in M_{2}$, and $a \in A$. 
The algorithm proposed here consists in first computing the modular decomposition of the graph, using existing linear time algorithms (see [16]). So we know if the graph contains a non-trivial module (which is a homogeneous set). If this preliminary search fails, we are lead to the case of searching proper homogeneous pairs in sesquiprime graphs. We thus assume now the graph is sesquiprime. Notice that $O(n+m)=O(m)$ as $G$ is connected.

Thanks to Remark 1 we have to search for a homogeneous pair $P$ that is not a module, i.e. such that $\left|M_{1}\right|>0$, and $\left|M_{2}\right|>0$, and $|A|+|C|>0$. We can assume, without loss of generality that $|A|>0$.

Proposition 14 Given $S=\left\{m_{1}, m_{2}, a\right\}$ a HP-seed set, every vertex $x \in V \backslash S$ has $|\operatorname{list}(x)|=2$.

Proof If $x \in N(a) x$ may belong to $M_{1}$ but not to $M_{2}$, and otherwise to $M_{2}$ but not to $M_{1}$. Whether $x$ is adjacent with $m_{1}$, or $m_{2}$, or both $m_{1}$ and $m_{2}$, or neither of them, then it may only belongs to (respectively) $A, C, B$ and $D . \square$

Given that, we can use our refinement method.

Lemma 3 If $G$ is prime, then in every proper homogeneous pair $\left\{M_{1}, M_{2}\right\}$ there exists a non-edge $\{x, y\} \notin E$ with $x \in M_{1}$ and $y \in M_{2}$

Proof Assume by contradiction that no such edge exists. $M_{2}$ would then be adjacent to $M_{1}$, thus both $M_{1}$ and $M_{2}$ would be modules of $G$, contradicting either the assumption of the primality of $G$ or the properness of $\left\{M_{1}, M_{2}\right\}$.

Proposition 15 Consider a vertex $z \in M_{2}$ such that $M_{1} \cap \overline{N(z)} \neq \varnothing$. Its non-neighborhood contains vertices from these four sets: $M_{1}^{\prime}=\overline{N(z)} \cap M_{1}$; $M_{2}^{\prime}=\overline{N(z)} \cap M_{2} ; A$; and $D$

Let us take such a vertex $z$. A spanning forest of $G[\overline{N(z)}]$ (obtained for example by a breadth first search) will necessarily contain one edge between a vertex of $A$ and a vertex of $M_{1}^{\prime}$, since it is the only way to go from $M_{1}^{\prime} \cup M_{2}^{\prime}$ to $A \cup D$. Indeed, $B \notin \overline{N(z)}$ and $C \notin \overline{N(z)}$, whereas $M_{1}^{\prime}$ and $A$ both contain at least one vertex (by Lemma 3 and assumption). Such spanning forest contains $O(n)$ edges, including at least one that will have one endpoint in $M_{1}$ and one endpoint in $A$. From the above discussion, we have:

Proposition 16 It is possible to generate $O\left(n^{2}\right) H P$-seed sets for the homogeneous pair problem, such that every proper homogeneous pair extends at least one of them.

This is implemented by Algorithm 3, who generates $O\left(n^{2}\right)$ triples to test, and runs in $O(m n)$ time.

Then we have, using $n^{2}$ times the EXTEND algorithm (where $H$ is the $2 \mathrm{x} 4$ matrix defining homogeneous pairs):

Theorem 7 There is a $O\left(m n^{2}\right)$ time algorithm for the homogeneous pair problem. 


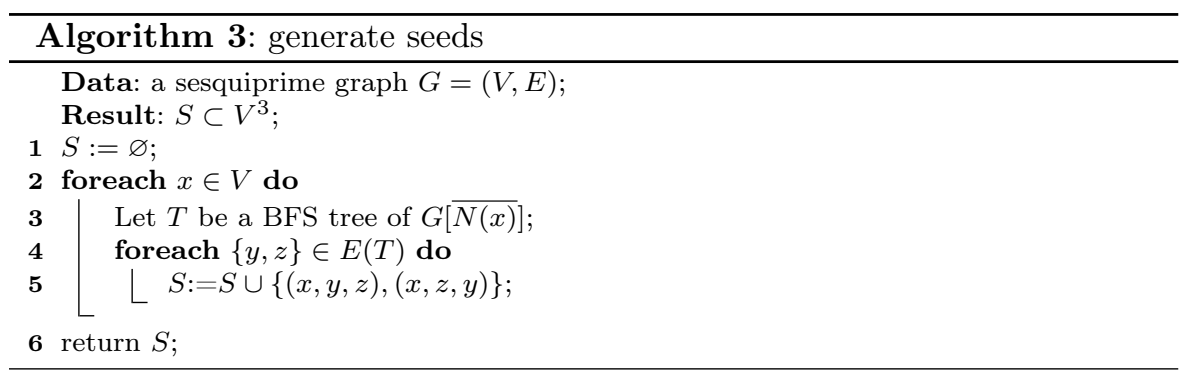

5.2 Finding a Maximal Homogeneous pair

Given two homogeneous pairs $P=\left\{M_{1}, M_{2}\right\}$ and $P^{\prime}=\left\{M_{1}^{\prime}, M_{2}^{\prime}\right\}$ we note $P \subset P^{\prime}$ for $\left(M_{1} \cup M_{2}\right) \subset\left(M_{1}^{\prime} \cup M_{2}^{\prime}\right)$.

Lemma 4 Let $P=\left\{M_{1}, M_{2}\right\}$ and $P^{\prime}=\left\{M_{1}^{\prime}, M_{2}^{\prime}\right\}$ be two homogeneous pairs with $P \subset P^{\prime}$.

1. If $\left(M_{1} \cup M_{2}\right) \subset M_{1}^{\prime}$, then $\left(M_{1} \cup M_{2} \cup A \cup C\right) \subseteq P^{\prime}$.

2. Else, $M_{1} \subseteq M_{1}^{\prime}$ and $M_{2} \subseteq M_{2}^{\prime}$; or $M_{1} \subseteq M_{2}^{\prime}$ and $M_{2} \subseteq M_{1}^{\prime}$.

Proof In the first case, since $P \subset M_{1}^{\prime}, A$ and $C$ are splitters of $M_{1}^{\prime}$. So by definition of a homogeneous pair, we have $A \in P^{\prime}$, and $C \in P^{\prime}$. If we are not in the first case, then we cannot have $M_{1}$ intersecting both $M_{1}^{\prime}$ and $M_{2}^{\prime}$; nor $M_{2}$ intersecting both $M_{1}^{\prime}$ and $M_{2}^{\prime}$, because then $A^{\prime}$ or $C^{\prime}$ would split $M_{1}$ or $M_{2}$. So we are in the second case.

Lemma 5 Given a non-trivial homogeneous pair $P_{i}=\left\{M_{1}^{i}, M_{2}{ }^{i}\right\}$, if there exists a non-trivial homogeneous pair $P_{i+1}=\left\{M_{1}^{i+1}, M_{2}^{i+1}\right\}$ and $\left(M_{1}^{i} \cup M_{2}^{i}\right) \subset$ $M_{1}^{i+1}$, then $P_{i+1}$ can be found in $O(m n)$ time.

Proof We are in the first case of Lemma 4. Create a set $M_{1}^{i+1}$ and affect to it all $M_{1}^{i} \cup M_{2}^{i} \cup A^{i} \cup C^{i}$ vertices. For each vertex $a \in N\left(M_{1}^{i+1}\right)$ try launching the REFINEMENT procedure using $A^{i+1}=\{a\}$ and $M_{2}^{i+1}$ as the splitters of $M_{1}^{i+1}$ that also belong to $\overline{N(a)}$. Indeed neighborhood of $a$ allows to split $P_{i+1}$. greedily adding the splitters of $P \cup A \cup C$ ends in finding some vertex of $M_{2}^{\prime}$. $\square$

Lemma 6 Given $x \in M_{1}, y \in M_{2}, z \in A$, and $t$ any other outer vertex as only member of $F$, procedure REFINEMENT returns $P$, a maximal homogeneous pair such that $x \in M_{1}, y \in M_{2}, z \in A$, and $t \in V \backslash P$.

Proof According to Proposition 14, all vertices are ambiguous, except $t$, the only vertex from Queue $F$. Proposition 4 rules only affect vertices to the side of the placed vertices used, so all placed vertices are on the side of $t$. Furthermore, the algorithm adds only necessary vertices to $A, B, C, D$ (by proposition 4 ). Then all remaining placed vertices are put on side $M_{1} \cup M_{2}$. $\square$ 
Lemma 7 Given a non-trivial homogeneous pair $P=\left\{M_{1}, M_{2}\right\}$, such that there is no non-trivial pair $P^{\prime}=\left\{M_{1}^{\prime}, M_{2}^{\prime}\right\}$ with $P \subset M_{1}^{\prime}$ or $P \subset M_{2}^{\prime}$, then a maximal non-trivial (with respect to inclusion) homogeneous pair $P^{\prime \prime}$ can be found in $O\left(m n^{2}\right)$ time.

Proof For any homogeneous pair $P^{\prime}$ containing $P$, we are in the second case of Lemma 4 . Try every pair $\{z, t\}$ of vertices of $V \backslash P$ as outer vertices, and launch the REFINEMENT procedure. One of the $O\left(n^{2}\right)$ found homogeneous pairs will be maximal, especially the larger one $P^{\prime \prime}$, thanks to Lemma 6. This step takes $O\left(m n^{2}\right)$ time as there are $O\left(n^{2}\right)$ calls to REFINEMENT

Theorem 8 A maximal non-trivial homogeneous pair of $G$ can be found in $O\left(m n^{2}\right)$ time

Proof We show it by constructing an sequence of homogeneous pairs $P_{0} \ldots P_{k}$ ordered by inclusion.

$P_{0}$ is built using Algorithm 1 with any seed $\left\{x \in M_{1}, y \in M_{2}, z \in A\right\}$ returning a non-trivial result. Lemma 6 (using as $t$ the $v_{0}$ of Algorithm 1) tells that at least one of the $O\left(n^{2}\right)$ tries of Proposition 16 should work, or the graph contains no non-trivial homogeneous pair.

Using Lemma 5 recursively, we get a sequence non-trivial of homogeneous pairs $P_{i}=\left\{M_{1}^{i}, M_{2}^{i}\right\}, 0 \leq i<k$, with $P_{i} \subsetneq M_{1}^{i+1}$. Notice $k \leq n$ since inclusion is strict so total time is $O\left(m n^{2}\right)$.

When this is no more possible, we complete this sequence with a final nontrivial pair $P_{k}$ found using Lemma 7, which is guaranteed by this Lemma to be maximal. Notice $k \leq n$ since inclusion is strict. $\square$

\subsection{Computing the Almost Maximal Homogeneous Pair Partition of a graph}

Lemma 8 Given a family of $O\left(n^{2}\right)$ homogeneous pairs containing all maximal homogeneous pairs, Algorithm 4 computes the AMHPP in $O\left(n^{3}\right)$ time.

Proof It is a simple implementation of Theorem 6. The array mark[ ] contains for each vertex either the number of its AMHP or $c$ if it is maximal conflicting. Sorting the homogeneous pairs in decreasing size order gives a linear extension of the inclusion order, therefore a maximal homogeneous pair is seen before all the ones it contains. At most two vertices of this pair then belong to already seen homogeneous pairs and they are marked as maximal conflicting. The running time is $O\left(n^{3}\right)$ (in fact it is linear w.r.t. input size).

Theorem 9 It is possible to find all almost maximal homogeneous pairs of a sesquiprime graph (i.e. the AMHPP) in time $O\left(m n^{2}\right)$

Proof Let $P$ be a maximal homogeneous pair found using Theorem 8 Let $\mathcal{S}$ be the set of $O\left(n^{2}\right)$ triples generated by Algorithm 3 . Define $\mathcal{S}^{-P}$ as the subset of triples of $\mathcal{S}$ so that at least one of their inner vertices does not belong to 


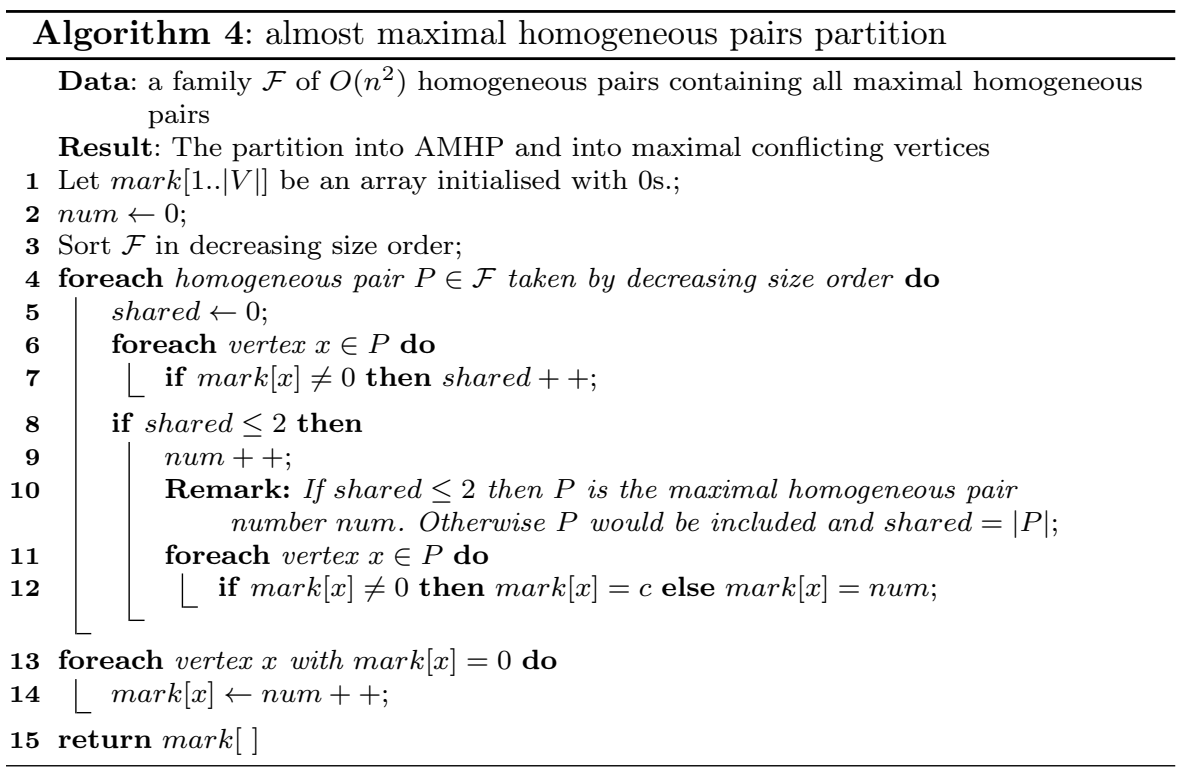

$P$, and so that they are compatible with at least one non-trivial homogeneous pair.

Theorem 5 tells that, for another maximal homogeneous pair $P^{\prime}$, it intersects $P$ on at most one vertex.

So for any two vertices $v_{0}$ and $v_{1}$ of $P$ we are sure one of them is out of the intersection, i.e. is in $V \backslash P^{\prime}$.

Thus, by calling REFINEMENT twice for any triple $(x, y, z) \in \mathcal{S}^{-P}$, once with $F=\left\{v_{0}\right\}$, and the second time with $F=\left\{v_{1}\right\}$, according to Proposition 6 we get a family $\mathcal{F}$ of $O\left(n^{2}\right)$ non-trivial homogeneous pairs excluding $P$ in $O\left(m n^{2}\right)$ time, each one being maximal with respect to its seed and to $v_{0}$ (or its seed and $\left.v_{1}\right) . \mathcal{F} \cup\{P\}$ therefore contains all maximal non-trivial homogeneous pairs of the graph.

Some of our homogeneous pairs might still not be maximal, but they will be properly included into one another. Thus, we only need to scan our homogeneous pairs again, deleting the ones that are included into others homogeneous pairs. This can also be done in $O\left(n^{3}\right)$ time using Algorithm 4 , and we get the AMHPP $\mathcal{M}$ of almost maximal homogeneous pairs of $G$. $\square$

\section{Conclusions and perspectives}

The algorithms described above share some common features and they are all particular cases of graph partition problems that can be expressed using matrices in $\{0,1, *\}$ as defined in [14. For many polynomial cases the techniques developed here can be used to speed up the existing algorithms. It is possible 
to patch the above algorithms to output possibly trivial $H$-joins, or to solve the list version of the problem we consider.

In this paper for the computation of non trivial $H$-join, we have carefully studied how to extend a seed set and our proposal is quasi optimal, but the other step : enumerating all potential seed sets, is far from being optimal. This could be improved, at least for some particular classes of graphs $H$, or by finding a better way to enumerate all the seed sets.

The structure of homogeneous pairs in sesquiprime graphs is now more undersood, leading to a canonical decomposition theorem. It is not clear however when the graph contains modules or sesquimodules.

\section{Aknowledgments}

The authors whish to thank the referees for their very careful readings, their suggestions greatly improve our writing.

\section{References}

1. P. Aboulker, P. Charbit, M. Chudnovsky, N. Trotignon and K. Vušković, LexBFS, structure and algorithms, CoRR, arxiv abs/1205.2535 (2012).

2. C. Aossey and K. Vušković, $3 P C(.,$.$) -free Berge graphs are perfect, Cited by Cornuéjols$ but seems unpublished.

3. B. Aspvall, M. F. Plass and R. E. Tarjan, A Linear-Time Algorithm for Testing the Truth of Certain Quantified Boolean Formulas, Inf. Process. Lett., 8(3):121-123, 1979.

4. B-M. Bui-Xuan, J. A. Telle and M. Vatshelle, H-join decomposable graphs and algorithms with runtime single exponential in rankwidth, Discrete Applied Mathematics, 158(7): 809-819, 2010.

5. B-M.Bui-Xuan, J.A.Telle and M.Vatshelle, Boolean-width of graphs, Theor. Comput. Science, 412(39): 5187-5204, 2011.

6. M. Conforti, G. Cornuéjols, A. Kapoor, and K. Vušković, Balanced 0, \pm 1 matrices, Part I: Decomposition theorem, and Part II: Recognition algorithm, Journal of Combinatorial Theory B 81 (2001), 243-306

7. W. H. Cunningham and J. Edmonds, A combinatorial decomposition theory, Canad. J. Math, vol 32(3): 734-765, 1980.

8. P. Charbit, F. de Montgolfier, M. Raffinot, A Simple Linear Time Split Decomposition Algorithm of Undirected Graphs, to appear in SIAM J. of Discrete Mathematics.

9. M. Chein, M. Habib and M.C. Maurer, Partitive hypergraphs. Discrete Mathematics 37(1): 35-50, 1981.

10. P. Charbit, M. Habib, N. Trotignon, and K. Vuskovic: Detecting 2-joins faster http: //arxiv.org/abs/1107.3977 to appear in J. of Discrete Algorithms.

11. V. Chvátal and N. Sbihi, Bull-free graphs are perfect, Graphs Combin. 3: 127-139, 1987.

12. H. Everett, S. Klein and B. Reed, An algorithm for finding homogeneous pairs, Discrete Applied Mathematics 72: 209-218, 1997.

13. T. Feder, P. Hell, D. Král and J. Sgallx, Two Algorithms for General List Matrix Partitions, SODA (2005) 870-876.

14. T. Feder, P. Hell, S. Klein and R. Motwani, List Partitions, SIAM J. Discrete Mathematics 16, (2003) 61-80.

15. M. Habib, A. Mamcarz and F. de Montgolfier, Algorithms for some H-join decompositions, LATIN, LNCS N 7256, 446-457, 2012.

16. M. Habib and C. Paul, A survey of the algorithmic aspects of modular decomposition, Computer Science Review, 4: 41-59, 2010. 
17. M. Habib, C. Paul, and L. Viennot. Partition refinement techniques: an interesting algorithmic toolkit. International Journal of Foundations of Computer Science 10 (2): 147-170, 1999.

18. F. de Montgolfier, Décomposition modulaire des graphes: théorie, extensions et algorithmes, PhD. thesis, Université Montpellier 2, 2003.

19. R. Paige and R. E. Tarjan, Three Partition Refinement Algorithms, SIAM J. Computing 16: 973-989, 1987.

20. M. Rao, Décompositions de graphes et algorithmes efficaces $\mathrm{PhD}$. thesis, Université de Metz. 